dass hier allein - trotz grundsätzlicher Anwendbarkeit - die Vermutung aus der statistischen Fallkostenprüfung nicht ausreicht, um direkt eine Unwirtschaftlichkeit der SSBVerordnungen zu begründen.

Die Definition der Angemessenheit des verordneten Sprechstundenbedarfs gemäß $\ 2$ Abs. 2 der Sprechstundenbedarfsvereinbarung schließt den medizinisch notwendigen Mehrbedarf über die Zuordnung zu einer Leistungsposition hinaus - auch bei Beachtung der angezeigten Sorgfalt zum Patientenwohl - mit ein. Damit wird letztlich bei Wahrung der Angemessenheit die Wirtschaftlichkeit der Mehrbedarfsverordnung begründet. [...]

DOI: 10.1007/s00350-014-3904-z

\section{Anmerkung zu SG Dortmund, Urt. v. 22.1.2014 - S 9 KA 190/11}

\section{Thorsten Süß}

Die rechtskräftige Entscheidung ist begrüßenswert, aber auch mutig. Sie ergänzt die bundessozialgerichtliche Rechtsprechung. Dabei treten auch Widersprüche zur gefestigten Meinung des BSG auf. Bei näherer Betrachtung lösen sich diese allerdings auf.

Der Kläger griff den Regressbescheid des beklagten Beschwerdeausschusses mit der Begründung an, in seiner Praxis lägen Besonderheiten vor, die einen erhöhten Bedarf an bestimmten Kathetern erklärten. Er versorge viele Alten- und Pflegeheime, wo Katheter überdurchschnittlich häufig zum Einsatz kämen und zudem die Anlage überdurchschnittlich häufig frustran verlaufe. Für die Versorgung eines Patienten seien mehrere Anlageversuche notwendig, sodass ein erhöhter Mittelverbrauch vorliege, dem aber nur eine abrechenbare Leistung gegenüberstünde. Im folgenden Regressverfahren zogen sich die Prüfgremien auf die statistische Vergleichsmethode zurück. Die Zahl der Leistungen übersteige die Zahl der Verordnungen um mehr als das Doppelte. Darüber hinaus liege ein auffälliges Missverhältnis im Vergleich zu anderen Praxen derselben Fachrichtung vor.

Die Eigenwilligkeit des Falls liegt darin, dass das Prüfgremium die Praxisbesonderheit des Klägers - Versorgung zahlreicher Altenheime - im Grundsatz anerkannte. Es argumentierte aber, der Kläger habe den genauen Umfang nicht substantiiert dargelegt. Er habe also nicht aufgezeigt, bei welcher Behandlung in welchem Umfang Katheter erforderlich geworden seien. Das Gremium setzte also letztlich willkürlich eine Grenze fest, bis zu der es Abweichungen akzeptieren wollte.

Dem ist das Sozialgericht in zwei Argumentationsschritten entgegengetreten. Es stellte nicht grundsätzlich die sta-

Rechtsanwalt Thorsten Süß,

Kanzlei Bergmann und Partner,

Hafenstraße 14, 59067 Hamm, Deutschland tistische Prüfmethode in Abrede, die auch vom Bundessozialgericht in ständiger Rechtsprechung gebilligt wird ${ }^{1}$. Wenn aber die Sprechstundenbedarfsvereinbarungen regelmäßig auf die „Angemessenheit“ (vgl. etwa $\$ 2$ Abs. 2 der Vereinbarung der KVWL; $\$ 4$ Abs. 9 KVBW) des rechnerischen Verhältnisses zwischen Behandlungszahl und verordneten Mitteln abstellen, dürften die Gremien nicht bei einem schlicht mathematischen Vergleich stehen bleiben. Vielmehr gebiete es die „Sorgfalt zum Patientenwohl“, medizinische Besonderheiten der Versorgung einzubeziehen. Derartige Besonderheiten können nach der gängigen Lehrmeinung als „Praxisbesonderheiten“ auf der zweiten Stufe der statistischen Vergleichsmethode - dem Vergleich mit anderen Arztpraxen - geltend gemacht werden. Das SG Dortmund scheint sie - unreflektiert, allerdings nah am Wortlaut der Vereinbarung - bereits auf der ersten Stufe zu berücksichtigen. Diese Rückkoppelung an den Text der Vorschrift ist begrüßenswert.

Weitreichender für die Regresspraxis ist aber die zweite Annahme des SG: Da das Prüfgremium die Besonderheiten der Tätigkeit des Klägers selbst für berücksichtigenswert hielt, könne es sich nicht darauf zurückziehen, von ihm einseitige Substantiierung zu erwarten. Vielmehr müsse es ,als eine Überprüfungspflicht des Prüfgremiums angesehen werden“, zu ermitteln, in welchem Umfang den Kläger eine besondere Versorgungssituation treffe. Verletze es diese Pflicht, sei keine ausreichende Sachverhaltsermittlung vorhanden, um einen Beurteilungsspielraum zu eröffnen Diese Formulierung steht in einem scheinbaren Widerspruch zu der häufig anzutreffenden Ansicht, dass den Arzt die Darlegungs- und Beweislast für Praxisbesonderheiten treffe $^{2}$. In der Tat geht auch das BSG in ständiger Rechtsprechung davon aus, dass die Sozialbehörden zwar an den Amtsermittlungsgrundsatz aus $\$ 20$ SGB X gebunden sind. Die Berücksichtigung von Praxisbesonderheiten setze aber voraus, dass der Arzt substantiierte Umstände darlege ${ }^{3}$. Der Widerspruch zum Urteil des SG Dortmund lässt sich aber auflösen. Denn dadurch, dass der Arzt die Besonderheiten behauptet und das Gremium sich die Behauptung zu Eigen gemacht hat, liegt das übliche Regel-Ausnahme-Verhältnis nicht vor. Es hätte überhaupt eine Ermittlung des Sachverhalts stattfinden müssen. An dieser hätte der Kläger selbstverständlich mitwirken müssen und können. Das Prüfgremium hatte dem Kläger aber nicht einmal abverlangt, seinen Vortrag näher zu konkretisieren. Die schlichte Behauptung mangelnder Substantiierung im späteren Bescheid oder gar erst im Klageverfahren konnte der Amtsermittlungspflicht daher nicht genügen.

1) BSG - B 6 KA 46/99; B 6 KA 24/03 -, MedR 2004, 577; vgl. auch Altmiks, in: Bergmann/Pange/Steinmeyer (Hrsg.), NomosKommentar Medizinrecht, 2. Aufl. 2014, \$106 SGB V, Rdnrn. $51 \mathrm{ff}$.

2) Schroeder=Printzen, in: Ratzel/Luxenburger (Hrsg.), Handbuch Medizinrecht, 2. Aufl. 2011, \$7, Rdnr. 903.

3) BSG - B 6 KA 66/00 -; vgl. auch Altmiks, in: Bergmann/Pange/ Steinmeyer (Hrsg.), NomosKommentar Medizinrecht, 2. Aufl. 2014, 』106 SGB V, Rdnr. 58. 